\title{
Influence of Thermal Stresses on the Aeroelastic Stability of Supersonic Wings
}

\author{
M. A. BIOT* \\ Cornell Aeronautical Laboratory, Inc.
}

\section{SUMMAR Y}

The theory of static aeroelastic stability of supersonic wings including chordwise bending, as developed in reference 1 , is further extended to include the influence of stresses arising from thermal gradients. The wing spanwise twist distribution is found to obey an ordinary differential equation of the fourth order whose coefficients depend on the thermal gradients. The influence of finite deformation is taken into account by the fact that the coefficients of the differential equation also depend on the amplitude of the deformation. The thermal stresses affect the stability in two distinct and independent ways, through its influence on the effective torsional stiffness and on the anticlastic effect. These factors act in opposite dircctions in such a way that if the thermal stress produces an increase in torsional stability it decreases the anticlastic stability, and vice versa. It is possible to embody the effect of the thermal stresses in a single parameter. Stability curves constitute a single family in this thermal parameter and plots are shown for three numerical values of the parameter.

\section{Evaluation of the Membrane Strress}

$\mathbf{I}$ N REFERENCE 1 we have investigated the aeroelastic stability of supersonic wings including chordwise bending. It was found that the stability is extremely sensitive to the anticlastic effect-i.e., the tendency of the wing to acquire a saddle shape as it deflects. It must be expected that thermal stresses have a strong influence on the anticlastic effect and therefore also on the aeroelastic stability. Our purpose here is to give a simplified treatment, leading to an evaluation of the influence of thermal stresses on the wing stability, including the chordwise bending, the anticlastic effects, the change in effective torsional stiffness, and the nonlinear aspects due to finite deformation.

We follow the procedure developed in Section 7 of reference 1 . This is a modified strip method which takes into account the influence of Poisson's ratio along with the membrane stresses. We consider a cantilever wing of rectangular plan form and double-wedge airfoil section (see Fig. 1). The method, however, is not restricted to this particular configuration. For simplicity, we shall assume the temperature distribu tion to be parabolic along the chord and independent of the spanwise coordinate. As is pointed out below, this is an approximation which embodies the essential temperature parameter. The present treatment is easily extended to cases of nonuniform distribution along the span by introducing a spanwise variation of this parameter. Also, as is pointed out below, the

Received September 5, 1956.

* Research Consultant. equivalent parameter for a nonparabolic distribution may be introduced by an averaging method.

We represent the chordwise temperature distribution by

$$
\begin{aligned}
\theta=\left\{\left[\left(\theta_{1}+\theta_{2}\right) / 2\right]-\theta_{0}\right\} & \left(x^{2} / l^{2}\right)+ \\
& {\left[\left(\theta_{2}-\theta_{1}\right) / 2\right](x / l)+\theta_{0} }
\end{aligned}
$$

where $\theta_{0}$ is the temperature at the midchord $(x=0)$ while $\theta_{1}, \theta_{2}$ are tempcratures, respectively, at the leading edge $(x=-l)$ and the trailing edge $(x=l)$. These temperatures are taken to be average temperatures across the thickness.

We denote by $\sigma_{x x}$ and $\sigma_{y y}$ the two stress components in the plane of the wing. It is easily shown by proceeding as in the theory of plates for large deformation ${ }^{6}$ that they satisfy the equation

$$
\nabla^{2}\left(\sigma_{x x}+\sigma_{y y}+\alpha_{t} E \theta\right)=-E \gamma
$$

where $\alpha_{t}$ (assumed constant) is the coefficient of thermal expansion and $\gamma$ is the Gaussian curvature of the deflected wing surface,

$$
\gamma=w_{y y} w_{x x}-w_{x y}^{2}
$$

The subscripts indicate partial derivatives $\partial^{2} / \partial x^{2}$, $\partial^{2} / \partial y^{2}, \partial^{2} / \partial x$ oy of the deflection $w$ taken positive upward in Fig. 1. We may assume the membrane stress to be only spanwise-i.e., $\sigma_{x x}=0$. Moreover, we may put

$$
\alpha_{t} \nabla^{2} \theta=\left(\alpha_{t} / l^{2}\right)\left[\theta_{1}+\theta_{2}-2 \theta_{0}\right]=\delta
$$

This is a quantity proportional to the chordwise curvature of the temperature distribution. It is the significant temperature parameter. Since we take $\sigma_{x x}=$ 0 , we write Eq. (2) as

$$
\partial^{2} \sigma_{y y} / \partial x^{2}=-E(\gamma+\delta)
$$

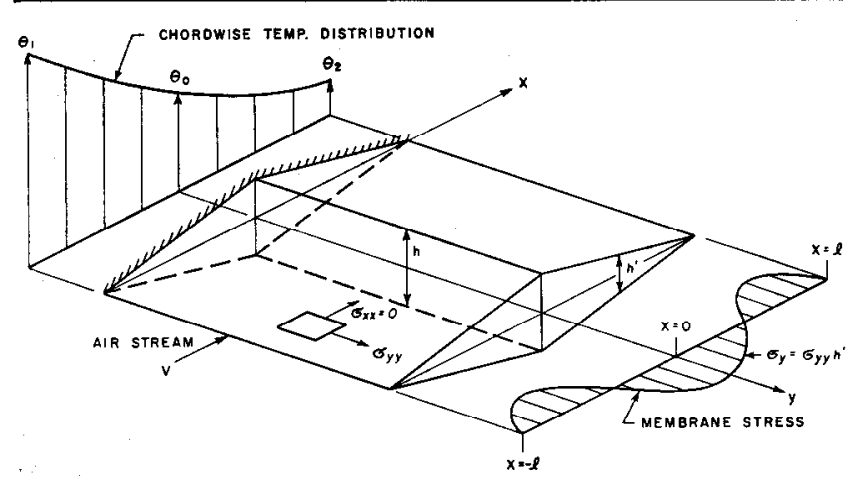

FIG. 1. General configuration of the wing, temperatures, and stresses. 
In first approximation we may assume the curvature $\gamma$ to be constant along the chord. We see immediately the reason for which we have assumed a parabolic variation of the temperature since this yields a constant value for $\delta$. The effect of the temperature then plays the same role as the curvature $\gamma$, and we may therefore proceed exactly as Section 7 of reference 1 . There is one difference, however, which constitutes an improvement. In the previous work we considered the total membrane stress - i.e., the stress $\sigma_{y y}$ integrated across the local thickness $h^{\prime}$,

$$
\sigma_{y}=\sigma_{y y} h^{\prime}
$$

and we assumed that it satisfied the approximate equation, Eq. (7.1) of reference 1,

$$
\partial^{2} \sigma_{y} / \partial x^{2}=-E h^{\prime} \gamma
$$

Actually it is more correct to use Fq. (5), and we shall rederive the theory on the basis of Eq. (5) instead of Eq. (6).

The distribution of the stress along the chord is obtained by integrating Eq. (5) and choosing the constants of integration in such a way that the total stress is self-equilibrating - i.e.,

$$
\int_{-l}^{+l} h^{\prime} \sigma_{y y} d x=0 \quad \text { and } \quad \int_{-l}^{+l} h^{\prime} \sigma_{y y} x d x=0
$$

The wing has a double-wedge cross section of maximum thickness $h$. Hence,

$$
h^{\prime}=h\left[1-\left|\frac{x}{l}\right|\right]
$$

This yields

$$
\sigma_{u y}=(1 / 2) E(\gamma+\delta)\left[\left(l^{2} / 6\right)-x^{2}\right]
$$

The chordwise distribution of the total membrane stress is

$$
\sigma_{\nu}=\frac{1}{2} E(\gamma+\delta) h l^{2}\left(1-\frac{x}{l} \mid\right)\left(\frac{1}{6}-\frac{x^{2}}{l^{2}}\right)
$$

\section{Differential EQuation for the Wing Twist}

We now evaluate the torque, acting on a strip of spanwise coordinate $y$. The local wing deflection is approximately

$$
w^{\prime}=w-\alpha x
$$

where $w$ and $\alpha$ are, respectively, the deflection and angle of attack at the midchord.* The local spanwise curvature is

$$
w_{y y^{\prime}}=w_{y y}-x \alpha_{y y}
$$

where $\alpha_{y y}$ is the second spanwise derivative of $\alpha$. The wing considered as a thin plate deflects under an equivalent load per unit area equal to

$$
q^{\prime}=\sigma_{y} w_{y y^{\prime}}-q
$$

* Note that $w$ is user for $w^{\prime}$ in the previous section. In the equations below, $w_{y y}, w_{x x}$, and $w_{x y}$ designate the second derivatives at the midchord. where $q$ is the aerodynamic load [see Eq. (1.1), reference 1]. Hence, from Eq. (11),

$$
q^{\prime}=\sigma_{u} w_{y y}-\alpha_{y y} \sigma_{y} x+q
$$

This load produces a torque, made up of two terms. One term $T^{\prime}$ is due to the load $-\alpha_{y y} \sigma_{y} x$, the other $T$ is an aerodynamic torque, originating with the chordwise curvature, which itself is caused by the load $q^{\prime}$. We shall first evaluate $T^{\prime}$. We find

$$
T^{\prime}=2 \alpha_{y y} \int_{0}^{l} \sigma_{y} x^{2} d x=\frac{-7}{360} E(\gamma+\delta) h l^{5} \alpha_{y y}
$$

The torque is positive in the stalling direction.

The chordwise curvature $w_{x x}$ produced by the load $q^{\prime}$ is obtained as in reference 1 by a method of virtual work, and by assuming that $w_{x x}$ is constant along the chord. We derive

$$
\begin{array}{r}
w_{x x}+v w_{y y}=-(7 / 30)\left(1-\nu^{2}\right) w_{y y}(\gamma+\delta) \times \\
\left(l^{4} / h^{2}\right)+(2 / 3)(k / l) \alpha
\end{array}
$$

In this expression, $\nu$ is Poisson's ratio and $k$ is the stability parameter defined in reference 1 as

$$
k=24\left(M^{2} / \sqrt{M^{2}-1}\right)\left[\rho c^{2}\left(1-\nu^{2}\right) / E\right](l / h)^{3}
$$

Eq. (15) is the same as Eq. (7.8) of reference 1, except for the addition of a temperature parameter $\delta$ and the numerical coefficient $7 / 30$ instead of $11 / 15$. The latter difference is due to our use of Eq. (5) instead of Eq. (6) for the membrane stress distribution. We must remember that $\gamma$ contains $w_{x x}$, hence, solving Eq. (15) for $w_{x x}$, we obtain

$$
w_{x x}=-\nu_{1}^{\prime} f w_{y y}+(2 / 3) f(k / l) \alpha
$$

$$
\text { with } \begin{aligned}
\nu_{1}{ }^{\prime} & =\nu-(7 / 30)\left(1-\nu^{2}\right)\left(l^{4} / h^{2}\right)\left(w_{x y}{ }^{2}-\delta\right) \\
f & =1 /\left[1+(7 / 30)\left(1-\nu^{2}\right)\left(l^{4} / h^{2}\right) w_{y y}{ }^{2}\right]
\end{aligned}
$$

The coefficient $\nu_{1}^{\prime} f$ is an "effective" Poisson's ratio which gives an immediate evaluation of the magnitude of the anticlastic effect associated with a spanwise bending $w_{y y}$. The effective Poisson's ratio is increased if $\delta$ is positive-i.e., if the temperature at the midchord is lower than the average of the leading- and trailingedge temperatures. We shall discuss the magnitude of this effect later.

The chordwise curvature $w_{x x}$ produces an aerodynamic torque which is easily evaluated. Its value (positive in the stalling direction) is

$T=(4 / 3)\left(M^{2} / \sqrt{M^{2}-1}\right) \rho c^{2} l^{2}\left[(2 / 3) f k \alpha-\nu_{1}^{\prime} f l w_{y y}\right]$

The total torque being $T^{\prime}+T$, we may writc a diffcrential equation for the spanwise distribution of the wing twist

$$
-Q\left(d^{2} \alpha / d y^{2}\right)=T^{\prime}+T
$$

where $Q$ is the wing torsional stiffness. Substituting the value of $T^{\prime}$ from Eq. (14), we may write

$$
-Q_{1}\left(d^{2} \alpha / d y^{2}\right)=T
$$


with an "effective" torsional stiffness

$$
Q_{1}=Q-(7 / 360) E(\gamma+\delta) h l^{5}
$$

We see immediately that if $\delta$ is positive-i.e., if the distribution of temperature is concave upward-its effect is to lower the effective torsional rigidity. The effect of the twist $w_{x y}$ alone appears through $\gamma=$ $-w_{x y}{ }^{2}$ which gives a measure of the increase of torsional rigidity due to finite twist. Torsional instability occurs for $Q_{1}=0$. For infinitesimal deformation, $\gamma=0$, and this gives the stability criterion in twist,

$$
\delta=(360 / 7)\left(Q / E h l^{5}\right)
$$

When $\delta$ exceeds this value a finite $\gamma$ appears, the magnitude of which is again derived from the condition $Q_{1}=0$-i.e.,

$$
-\gamma=\delta-(360 / 7)\left(Q / E h l^{5}\right)
$$

As mentioned above, the effect of the temperature on the aeroelastic stability appears, not only through a change in effective torsional rigidity, but also through the anticlastic effect. To evaluate the combined effect, we may proceed entirely as in Section 7 of reference 1 , provided we replace $Q$ by $Q_{1}$, and $\nu^{\prime}$ by $\nu_{1}{ }^{\prime}$. We write

$$
\begin{gathered}
P_{1}=h^{3} l E / 24\left(1-\nu^{2}\right) Q_{1} \\
R=h^{3} l / 6\left(1-\nu^{2}\right) I_{1}
\end{gathered}
$$

where $I_{1}$ is the wing cross section moment of inertia about the chord. Furthermore, we put

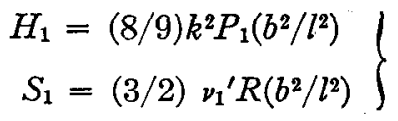

where $b$ is the wing span.

The differential equation for the wing twist in terms of a nondimensional spanwise coordinate $\eta=y / b$ is then identical with Eq. (7.17) of reference 1, except that $H$ and $S$ are replaced by $H_{1}$ and $S_{1}$-i.e.,

$$
\left(d^{4} \alpha / d \eta^{4}\right)+H_{1} f\left(d^{2} \alpha / d \eta^{2}\right)-f H_{1} S_{1} \alpha=0
$$

\section{Discussion}

The stability diagram in the $\sqrt{H_{1}}, S_{1}$ plane is the same as that given by Fig. 19 in the above reference. In order to discuss the effect of the temperature it is convenient to separate the parameter $\delta$ in the expressions of $H_{1}$ and $S_{1}$,

$$
H_{1}=H\left(Q / Q_{1}\right) \quad S_{1}=S\left(\nu_{1}{ }^{\prime} / \nu^{\prime}\right)
$$

$H$ and $S$ are defined as in reference 1 :

$$
\left.\begin{array}{rl}
H & =(8 / 9) k^{2} P\left(b^{2} / l^{2}\right) \\
S & =(3 / 2) \nu^{\prime} R\left(b^{2} / l^{2}\right) \\
P & =h^{3} l E / 24\left(1-\nu^{2}\right) Q \\
\nu^{\prime} & =\nu-(7 / 30)\left(1-\nu^{2}\right)\left(l^{4} / h^{2}\right) w_{x y^{2}}
\end{array}\right\}
$$

Because we have used Eq. (5) instead of Eq. (6) expressions for $\nu^{\prime}$ and $f$ are different from those of reference 1 . The temperature parameter is contained only in the factors $Q / Q_{1}$ and $\nu_{1}^{\prime} / \nu^{\prime}$. The influence of the

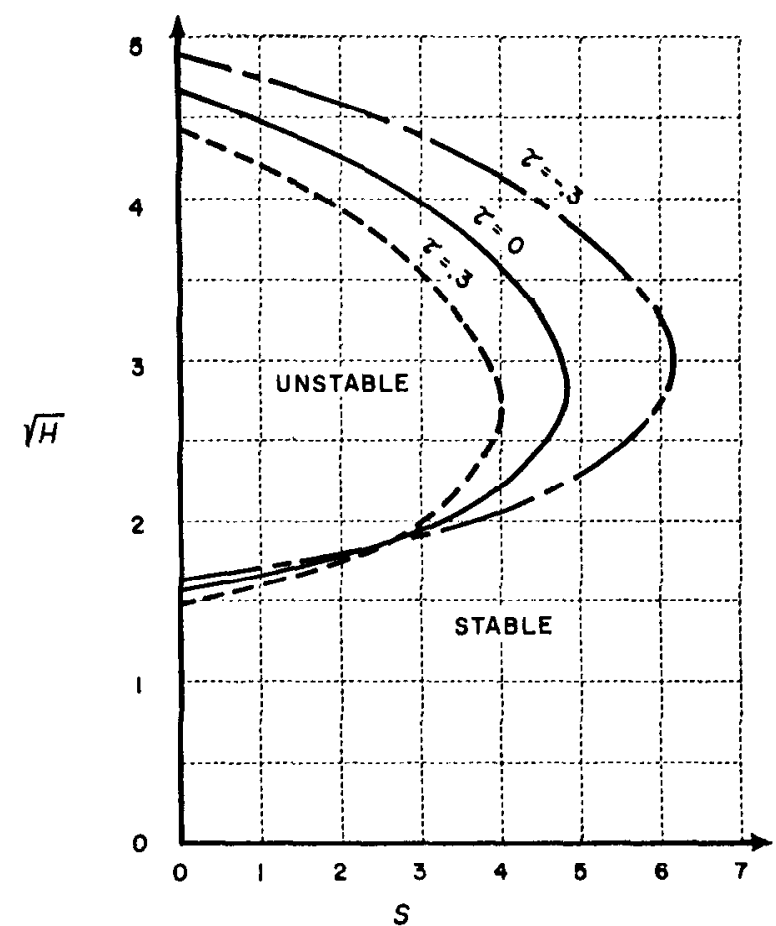

FIG. 2. Effect of the temperature parameter $(\tau)$ on the stability diagram.

temperature on the $\sqrt{H}, S$ stability diagram is therefore obtained by a change of scale of the coordinate axes. Consider, for instance, the case of incipient instability-i.e., small deformation-then $f=1, \nu^{\prime}=\nu$, $\gamma=0$. The torsional rigidity of the thin double wedge is

$$
Q=(1 / 6) l h^{3} G=(1 / 12)\left[l h^{3} E /(1+\nu)\right]
$$

hence (with $\nu=\nu^{\prime}$ and $w_{x y}=0$ )

$$
\left.\begin{array}{l}
Q_{1} / Q=1-(7 / 30)(1+\nu)\left(l^{4} \delta / h^{2}\right) \\
\nu_{1}^{\prime} / \nu^{\prime}=1+(7 / 30)\left[\left(1-\nu^{2}\right) / \nu\right]\left(l^{4} \delta / h^{2}\right)
\end{array}\right\}
$$

The influence of the temperature is contained in the nondimensional parameter

$$
\tau=l^{4} \delta / h^{2}=\alpha_{t}\left[\theta_{1}+\theta_{2}-2 \theta_{0}\right]\left(l^{2} / h^{2}\right)
$$

For steel we put $\alpha_{t}=7.22 \times 10^{-6} /{ }^{\circ} \mathrm{F}$. and $\nu=0.3$. If we assume $(1 / 2)\left(\theta_{1}+\theta_{2}\right)-\theta_{0}=208^{\circ} \mathrm{F}$. and $l / h=$ 10 , this case corresponds to $\tau=0.3$. Fig. 2 shows a plot of the diagram for three values $\tau=0.3, \tau=0$, and $\tau=$ -0.3 . The value of $\tau=-0.3$ corresponds to a midchord temperature in excess of the average temperatures of leading and trailing edge. This can happen during a cooling period of a heated wing.

Fig. 2 indicates that for positive $\tau$ the effect of the temperature is destabilizing with regard to torsional rigidity and stabilizing with regard to the anticlastic effect. An interesting result is that for negative $\tau$ (cooling phase) the influence of the temperature on the stability is reversed.

The present treatment can easily be extended to include spanwise variations of temperature and the effect of the wing tip and aspect ratio on the structural 
properties. Variational methods are suggested as a practical approach.

We note that in evaluating the effect of the temperature on the torsional rigidity, it is not necessary to assume a parabolic distribution of the temperature along the chord. A refinement in the present treatment is to introduce the equivalent value of $\delta$ giving the same torsional rigidity. Referring to Eqs. (14) and (21), we may define $Q_{1}$ as*

$$
Q_{1}=Q+2 \int_{0}^{l} \sigma_{y} x^{2} d x
$$

The stress $\sigma_{y}$ is the total membrane stress across the thickness.

* The influence of axial stress on torsional stiffness was previously investigated by Wagner, ${ }^{2}$ Biot, ${ }^{3}$ and Goodier. Simplified expressions were also derived by Budiansky and Mayers ${ }^{5}$ and by Hoff.?

\section{REFERENCES}

${ }_{1}$ Biot, M. A., The Divergence of Supersonic Wings Including Chordwise Bending, Journal of the Aeronautical Sciences, Vol 23, No. 3, pp. 237-251, March, 1956.

2 Wagner, H., Tension and Buckling of Open Sections, NACA TM 807, October, 1936.

${ }^{3}$ Biot, M. A., Increase of Torsional Stifness of a Prismatical Bar Due to Axial Tension, Journal of Applied Physics, Vol. 10, No. 12, pp. 860-864, December, 1939.

- Goodier, J. N., Elastic Torsion in the Presence of Initial Axial Stress, Journal of Applied Mechanics, Vol. 17, No. 4, p. 383, 1950.

5 Budiansky, B., and Mayers, J., Influence of Aerodynamic Heating on the Effective Torsional Stiffness of Thin Wings, Journal of the Aeronautical Sciences, Vol. 23, No. 12, p. 1081, December, 1956.

${ }^{6}$ Love, A. E. H., The Mathematical Theory of Elasticity, p. 558; Dover Publications, New York.

${ }^{7}$ Hoff, N. J., Approximate Analysis of the Reduction in Torsional Rigidity and of the Torsional Buckling of Solid Wings Under Thermal Stresses, Readers' Forum, Journal of the Aeronautical Sciences, Vol. 23, No. 6, p. 603, June, 1956. 